\title{
The Influence of Algal Exudate on the Hygroscopicity of Sea Spray Particles
}

\section{H. Wex, ${ }^{1}$ E. Fuentes, ${ }^{2}$ G. Tsagkogeorgas, ${ }^{1}$ J. Voigtländer, ${ }^{1}$ T. Clauss, ${ }^{1}$ A. Kiselev, ${ }^{1}$ D. H. Green, ${ }^{3}$ H. Coe, ${ }^{2}$ G. McFiggans, ${ }^{2}$ and F. Stratmann ${ }^{1}$}

\author{
${ }^{1}$ Leibniz-Institute for Tropospheric Research, Permoser Strabe 15, 04318 Leipzig, Germany \\ ${ }^{2}$ Centre for Atmospheric Sciences, University of Manchester, Manchester, M13 9PL, UK \\ ${ }^{3}$ The Scottish Association for Marine Science, PA37 1QA Oban, UK
}

Correspondence should be addressed to H. Wex, wex@tropos.de

Received 7 February 2010; Accepted 7 June 2010

Academic Editor: Chris Osburn

Copyright (c) 2010 H. Wex et al. This is an open access article distributed under the Creative Commons Attribution License, which permits unrestricted use, distribution, and reproduction in any medium, provided the original work is properly cited.

\begin{abstract}
We examined the effect of organic matter released by four different algal species on the hygroscopic growth and droplet activation behaviour of laboratory-generated marine aerosol particles. Hygroscopic growth factors and dry diameters for activation were reduced by less than $10 \%$, compared to that of sodium chloride or of artificial seawater that was devoid of marine surfactants. Concentration-dependent nonideal behaviour was observed for the artificial seawater. But within measurement uncertainty, the measured hygroscopic growth and droplet activation behaviour for the samples that contained organic matter were consistent with a hygroscopicity parameter that was constant between the sub- and supersaturated measurement points. Also, the hygroscopic growth measured for hydrated particles after 3 and after 10 seconds was similar, which implies that in this time range no kinetic effects were detected.
\end{abstract}

\section{Introduction}

Approximately $71 \%$ of the Earth's surface is covered by oceans, which provide a constant source of marine aerosol particles. Atmospheric marine aerosols consist of particulate matter with both, primary and secondary origin. Marine primary particles are produced on the ocean surface by bubble-bursting and tearing from breaking waves, that is, by processes depending on the wind speed. Secondary particulate matter originates from gas-to-particle conversion processes, such as nucleation and condensation [1], and, in the marine case, comprises substances as non-sea-salt sulphate and organic species.

Regarding the Earth's radiation budget, marine particles affect both the aerosol direct and indirect effects. Pilinis et al. [2] stated that the single most important parameter in determining direct aerosol forcing is the relative humidity $(\mathrm{RH})$, and the most important process is the increase of the aerosol mass as a result of water uptake. Compared to continental aerosol, marine aerosol particles generally are more hygroscopic (see e.g., [3], where the particle hygroscopicity parameter $\kappa$ [4] of the more hygroscopic particle fraction was determined to be around 0.3 for continental and 0.45 for marine aerosol, and with a $\kappa$ of 0.95 for an additional (small) sea-salt mode for the marine case). This makes marine aerosol particles particularly susceptible to changes in relative humidity. Likewise, they easily can be activated to cloud droplets at atmospheric relevant supersaturations. The largest sea-salt particles may behave as giant Cloud Condensation Nuclei (CCN), [5] and their role in the initiation of precipitation in warm shallow clouds is still under discussion (e.g. [6-8]).

Model results obtained by Textor et al. [9] showed that worldwide all-model-average emissions are dominated by the mass of sea-salt, followed by dust, non-sea-salt sulphate, particulate organic matter, and finally black carbon. Marine stratus and stratocumulus clouds contribute about $30 \%$ to $40 \%$ to the Earth's albedo $[10,11]$. Therefore, overall, marine aerosol particles can be assumed to play an important role for the Earth's atmosphere on a global scale.

The chemical compounds present in an aerosol particle influence its hygroscopic growth and its activation to a 
cloud droplet. The influence of organic material contained in marine particles on their hygroscopic growth and activation behaviour continues to be the subject of intensive investigation. Organic material in these particles can originate from different sources. For marine aerosol, one source is the metabolic activity of oceanic phytoplankton which produces a complex mixture of dissolved and particulate species, including organic compounds such as carboxylic acids, lipids, amino acids, and carbohydrates [12]. These components can become incorporated into particles during the bubble-bursting process. A further source for organic material in marine aerosol is of secondary nature; volatile gases (e.g., isoprene produced by phytoplankton, but also gases emitted by the terrestrial biosphere or such of anthropogenic origin) can be precursors for substances condensing onto the existing particles $[13,14]$.

Different studies have examined marine aerosol with respect to its content of organic matter. Randles et al. [15] assumed this content to constitute up to $50 \%$ of the dry submicron marine aerosol mass. Cavalli et al. [16] found water-soluble and water-insoluble organic carbon (WSOC and WINSOC, resp.) in samples of marine aerosol collected during a phytoplankton bloom period in the North Atlantic. WINSOC was found to be the dominant submicron and the second dominant supermicron aerosol species (only exceeded by sea-salt), followed by WSOC as the next abundant species in both cases. The WSOC comprised about $20 \%$ to $30 \%$ of the total carbon and consisted of aliphatic and partially oxidized species and humic-like substances. The organic fraction found by Cavalli et al. [16] was reported to feature appreciable surface-active properties, where the bulk surface tension reduction could be expressed in terms of the concentration of dissolved organic carbon using the Szyszkowsky-Langmuir expression. Results from Cavalli et al. [16] agree with those described in [17], where North Atlantic marine aerosol arriving at the west coast of Ireland (Mace Head) was analyzed. O'Dowd et al. [17] observed that organic matter in the aerosol occurred in correlation with biological activity in the ocean. They also observed an increase in the organic fraction with decreasing size. Among the analyzed WSOC from the particles, they found partly oxidized species which, they assume, could potentially cause large decreases in surface tension.

Mochida et al. [18] examined marine aerosol from the North Pacific and found that concentrations of lower molecular weight (C14 to C19) saturated fatty acids correlated with sea-salt concentration and occurred in correspondence to algal bloom periods. They concluded that the fatty acids were released from the ocean surface to the atmosphere, and they also stated that fatty acids are among the substances that are potentially filmforming and that could, therefore, influence the behaviour of the particles upon hydration.

Tervahattu et al. $[19,20]$ analyzed atmospheric particles of marine origin using aerosol mass spectrometry and electron microscopy and found fatty acids present in the particles. The possible influence of the fatty acids on the particles was discussed, and two opposing effects were mentioned. One of the discussed effects was the formation of a hydrophobic organic film that could act as a barrier to hygroscopic growth, a possibility that already earlier has been discussed in connection to sea-spray generated aerosols by Ellison et al. [21]. The second effect was a lowering of the particle surface tension, which in turn would facilitate the activation to a cloud droplet, as discussed in [22]. Such a surface tension reduction has been found in seawater samples that were enriched in organic matter through desalting by Moore et al. [23]. The carbon concentration at which a reduction of the surface tension of $1 \%$ (compared to that of water) was found was in the range from 300 to $800 \mathrm{mg} / \mathrm{L}$, which is above that naturally observed in seawater.

Sellegri et al. [24] examined the influence of a synthetic surfactant (SDS) on the particle generation from artificial seawater in the laboratory, using bubble-bursting processes to produce the particles. They observed from their measurements that both temperature and the surfactant concentration in the seawater influence the produced aerosol and its size distribution. They compared the obtained particle number size distributions to those typically measured for North Atlantic marine aerosol. This comparison is generally limited due to the possible addition of organic particulate matter from the gas phase to the marine particles. But Sellegri et al. [24] still suggest that marine submicron primary aerosol modes may mainly result from bubble bursting processes on a first approximation.

A modeling study by Randles et al. [15] showed that the direct aerosol effect is affected by the large amount of organic matter that can be contained in marine aerosol particles, in that this direct effect is noticeably diminished when the organic fraction is taken into account, compared to assuming the particles to consist of pure $\mathrm{NaCl}$. This is largely due to the hygroscopicity of the organic matter being lower than that of $\mathrm{NaCl}$. Additionally, as described above, chemical compounds that were said to potentially influence the surface tension of aerosol particles have been identified in marine samples. It is known that a significant suppression of surface tension has a large effect particularly on particle activation (e.g., [25]). Furthermore, solutions of $\mathrm{NaCl}$ and seawater samples are known to show a concentration-dependent nonideal behaviour, that is, the degree of nonideality changes with the concentration of the solute in the particle/droplet $[26,27]$. It is pertinent to ask whether hygroscopic growth and activation of multicomponent aerosol particles representative of those in the marine atmosphere may be described by a simple approximation to the Köhler equation or whether nonideality variation or the presence of surface active components invalidate such an approach.

To further elucidate this question, the present study examines the behaviour of seawater enriched by algal exudate. The algae species that were cultured to obtain algal exudates were Chaetoceros sp. (Chaet), Emiliana huxleyi (E. huxleyi), Phaeocystis (Phaeo), and Thalassiosira rotula (T. rotula). Chaet and T. rotula both belong to the group of diatoms which is one of the most common types of phytoplankton. E. huxleyi occurs in great abundance in the Earth's oceans, from the tropics to subarctic waters [28]. The Phaeocystis specie, too, is widely distributed throughout the world's oceans and is even found in sea ice. It can form floating colonies with hundreds of cells embedded in a 
polysaccharide gel matrix that can multiply massively during blooms [29]. These algae species are all major contributors to primary production of organic matter in the ocean, having an important role in marine food chains and in the global carbon cycle.

A bubble-bursting generator described in Fuentes et al. [30] was used in our study to generate particles from samples of artificial seawater and from samples containing additional organic material, that is, algal exudate from cultures of the above mentioned algae species. Size selected particles were used to measure hygroscopic growth and activation of the particles. The different data sets were used to derive particle hygroscopicities for a wide range of water activities, where we used the hygroscopicity parameter $\rho_{\text {ion }}$ as defined in [31].

\section{Theory}

The main goal of this study was to examine the hygroscopic behaviour of particles that consisted of salts contained in seawater and additional organic matter originating from algae. Hygroscopic diameter growth factors and critical dry diameters for activation were interpreted in terms of particle hygroscopicity. For that, the Köhler theory, which connects particle size and hygroscopicity to hygroscopic growth and activation, was used. The theory connects water vapor saturation at the surface of the droplet, $S_{d}$, to the Kelvin term accounting for the curvature of the droplet, $K$, and the water activity or Raoult term accounting for dissolved matter in the solution, $a_{w}$

$$
S_{d}=K a_{w}=\exp \left(\frac{4 M_{w} \sigma_{s}}{R T \rho_{w} d_{d}}\right) * \exp \left(-\frac{\phi v \rho_{s} V_{s}}{M_{s} n_{w}}\right),
$$

with the molecular weight of water $M_{w}$, the surface tension of the solution $\sigma_{s}$, the ideal gas constant $R$, the temperature $T$, the density of water $\rho_{w}$, and the diameter of the hydrated particle/droplet $d_{d}$, the osmotic coefficient $\phi$ (accounting for nonideality of the solution), the number of ions per solute molecule $\nu$, the solute density $\rho_{s}$, the volume of the solute (i.e., of the dry particle), $V_{s}$, the solute molecular weight $M_{s}$, and the number of water molecules in the droplet $n_{w}$.

The solute parameters of the Raoult term can be combined into a single hygroscopicity parameter, $\rho_{\text {ion }}[31]$ :

$$
\rho_{\text {ion }}=\frac{\phi v \rho_{s}}{M_{s}} .
$$

This approach is useful when the solute properties are unknown, as often is the case for organic compounds or for mixtures. If the properties of all the components in a mixture are known, then $\rho_{\text {ion }}$ can be obtained by applying a volume mixing rule. This will be used later in this study for an artificial seawater sample. Values of $\rho_{\text {ion }}$ are generally comparable to $\kappa$ as defined in [4]. As explained in [32], at $a_{w}>0.95, \rho_{\text {ion }} M_{w} / \rho_{w}=\kappa$ to better than $3 \%$.

The osmotic coefficient included in $\rho_{\text {ion }}$ is often assumed constant for dilute solutions such as at the high $\mathrm{RH}$ values considered in this study, that is, a possible change in nonideal solution behaviour with the concentration of the solute in the particle/droplet solution is omitted. This results in a constant value for $\rho_{\text {ion. }}$. However, it is well known that, for example, $\mathrm{NaCl}$ or ammonium sulphate show a concentration-dependent nonideal behaviour (e.g., [26]). When the nonideality of a solution in dependence of the solute concentration (i.e., the component activity coefficient) is known, a concentration-dependent description of $\rho_{\text {ion }}$ is possible. Or, vice versa, measurements can be made at different concentrations (e.g., by measuring hygroscopic growth at different relative humidities (RHs)), and if $\rho_{\text {ion }}$ derived from these measurements shows a concentration dependence, this could indicate a concentration-dependent nonideal behaviour.

We will make use of this in evaluating the influence of algal exudates on the hygroscopic behaviour of seawater in this work. In the analysis, $\rho_{\text {ion }}$ as derived from measured hygroscopic growth and activation (through use of (1) and (2)) will be employed.

\section{Sample Preparation}

Data from six different samples will be shown in this study. One sample was artificial seawater devoid of organics, one algae medium (natural seawater with nutrients), and four samples were seawater proxies containing mixes of exudates produced by four different algae species, namely Chaetoceros sp. (Chaet), Emiliana huxleyi (E. huxleyi), Phaeocystis (Phaeo), and Thalassiosira rotula (T. rotula).

The artificial seawater was prepared from analytical grade salts and deionised water following the method described by Kester et al. [33]. The ionic mass ratios of $\mathrm{Na}^{+}, \mathrm{Cl}^{-}, \mathrm{Mg}^{2+}$, $\mathrm{HCO}_{3}{ }^{-}$, and $\mathrm{SO}_{4}{ }^{2-}$ were comparable to those in seawater. The salt mixture contained, by mass, $73.6 \% \mathrm{NaCl}, 14.5 \%$ $\mathrm{MgCl}_{2}, 11.5 \% \quad \mathrm{Na}_{2} \mathrm{SO}_{4}$, and $0.4 \% \quad \mathrm{NaHCO}_{3}$. Seawater samples of $3.5 \%$ salinity were prepared by adjusting the solution density at the laboratory temperature using a hydrometer. This corresponds to concentrations of the salts in the artificial seawater of $22.9 \mathrm{~g} / \mathrm{L} \mathrm{NaCl}, 4.6 \mathrm{~g} / \mathrm{L} \mathrm{MgCl}_{2}$, $3.6 \mathrm{~g} / \mathrm{L} \mathrm{Na}_{2} \mathrm{SO}_{4}$, and $0.01 \mathrm{~g} / \mathrm{L} \mathrm{NaHCO}_{3}$.

Algal growth medium was prepared based on natural seawater from the North Atlantic (Tiree passage, Scotland, UK) that had been filtered with GF/C (Whatman), amended with F/2 nutrients and either selenite or silicate [34], and sterilized by autoclaving $\left(121^{\circ} \mathrm{C}\right.$ for $\left.15 \mathrm{~min}\right)$.

For the preparation of the different seawater proxies enriched with biogenic organics, the filtered natural seawater amended with algal medium was used for growing four different phytoplankton cultures in the laboratory. The algae cultures were grown in glass Erlenmeyer flasks. Monitoring of algal growth was done by measuring in vivo chlorophyll-a fluorescence every 2 days using a Turner Trilogy fluorometer. Following algae growth, the cell biomass was removed by filtration. Details of the culturing and subsequent filtering procedures can be found in [30].

Artificial seawater devoid of organics and natural seawater enriched with the produced biogenic matter were combined to prepare seawater proxies at $512 \mu \mathrm{M}$ DOC (Dissolved Organic Carbon), where DOC analysis was done using a Shimadzu TOC-V CPH/CPN. The samples were prepared 
such that one of the algal exudates comprised $50 \%$ of the total organic concentration, while the rest of the organic content was equally divided between the other three algal exudates on study (i.e., 16.7\% of total DOC for each algal exudate). The samples were named after the algae species which was the most abundant (e.g., the organic fraction in E. huxleyi Mix consisted to $50 \%$ of E. huxleyi exudate and to $16.7 \%$ of each of the other three algal exudates). The organic concentration selected (about $0.01 \mathrm{~g} / \mathrm{L}$ ) is within the range of concentration expected for organics in naturally enriched seawater in areas of high biological activity [35, 36]. This value for the organic concentration was derived from the adjusted value of $512 \mu \mathrm{M}$ DOC by considering the phytoplankton average elemental composition following Fraga [37], which then yields a molar mass of $2.38 \mathrm{kDa}$ and a molecule to $\mathrm{C}$ mass ratio of 1.87 .

The samples (each about $6 \mathrm{~L}$ in total) were kept frozen until shortly (some hours) before measurements took place, when they were left to thaw at laboratory temperature.

\section{Experimental Setup}

A bubble-bursting aerosol generation method, using the recirculation of liquid samples by means of a peristaltic pump, was used to produce a marine aerosol proxy (see Figure 1). Experiments were performed using a PTFE tank (internal dimensions: $20.5 \mathrm{~cm}$ height, $19.5 \mathrm{~cm}$ width, and $27.5 \mathrm{~cm}$ length) filled with $3.5-6 \mathrm{~L}$ of sample. The sample was recirculated and impinged on the sample surface in the tank. With this technique, air entrained in the sample by water impingement was dispersed in a rising plume of bubbles that burst at the sample surface. In order to generate a statistically significant number of particles for the aerosol experiments, the recirculating flow was divided in eight separate jets by using a flow distributor. The bubble plume penetration distance was about $5-7 \mathrm{~cm}$. Whilst bubble paths and lifetimes in the small-scale tank employed in this study were shorter than those expected in oceanic conditions [30], theoretical analysis of the kinetics of adsorption of marine organics on rising bubbles indicated that the time required for adsorption equilibrium is rapid compared to the characteristic bubble lifetimes in small-scale systems [30]. Hence, the experimental configuration employed should be valid for conducting studies on the effects of biogenic organics on the sea spray aerosol.

A chemically resistant Teflon composite tube (Masterflex $\mathrm{I} / \mathrm{P}$ 70) was employed for water recirculation in order to avoid contamination of the samples and adhesion of surfactants on the tubing wall. The water flow $(4 \mathrm{Lpm})$ was controlled by setting the peristaltic pump rotating speed to 35-40 rpm and monitored using a rotameter.

Thorough cleaning procedures were applied in order to minimize the presence of contaminants in the system. The tank walls and bubbling apparatus were rubbed with chloroform and isopropanol, rinsed and washed with deionised water. Deionised water was recirculated for 30-60 min in order to rinse the pump tubing prior the experiments.

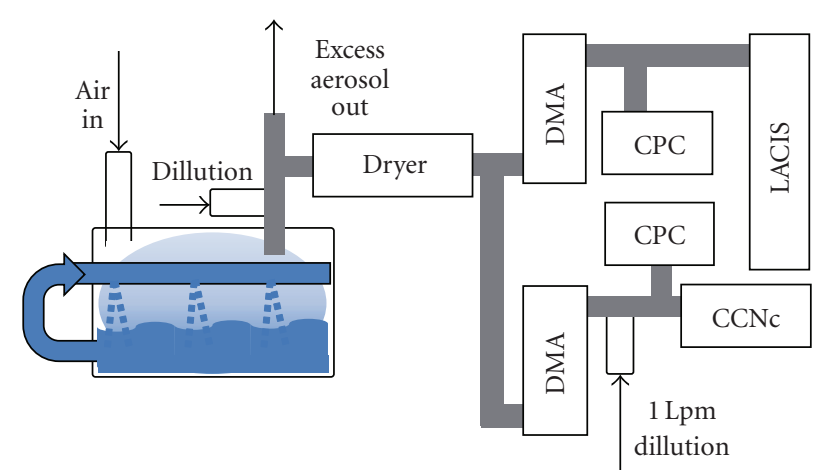

FIGURE 1: Sketch of the experimental setup.

For the aerosol experiments, the PTFE tank was sealed and swept with a continuous particle-free air flow of 33.5 Lpm. The humidity of the aerosol flow extracted from the outlet of the tank was reduced by dilution with dry, particlefree air (see Figure 1). The excess flow was vented through a T-connector, and the flow needed to feed the different instruments was led through a diffusion dryer, reducing the humidity to a value below $10 \% \mathrm{RH}$. After that, the dry aerosol flow was divided into two flows and distributed to the instruments.

In each of the two lines, dry particle sizes were selected by use of a neutralizer (Kr 85) and a Differential Mobility Analyzer (DMA) (Type Vienna Medium, aerosol to sheath flow rate of 1 to $10 \mathrm{Lpm})$. Downstream of each of the two DMAs, the particle number concentration of the quasi monodisperse aerosol were determined with a CPC (TSI3010 Condensation Particle Counter, TSI Inc., St. Paul Minnesota, USA).

In one line, the CPC measured with an aerosol flow of $0.9 \mathrm{Lpm}$, while the remaining $0.1 \mathrm{Lpm}$ was used for hygroscopic growth measurements with LACIS (Leipzig Aerosol Cloud Interaction Simulator, [38]). The number concentration measured with this CPC was only used to monitor the stability of the particle generation system. The size selected by the respective DMA, that is, the dry particle mobility diameter used for LACIS measurements was $200 \mathrm{~nm}$. For the measurements, the LACIS flow tube had a total length of $7 \mathrm{~m}$, and hygroscopically grown particles were detected at a tube length of $1 \mathrm{~m}$ and $7 \mathrm{~m}$, using two optical particle spectrometers (OPSs, [39]) simultaneously. Prior to the measurement campaign, the OPSs were calibrated with PSL-particles with diameters of 300, 400, 500, 700, 800, and $1600 \mathrm{~nm}$. The RH obtained in the LACIS flow tube was constantly controlled after each of the measurements by measuring the hygroscopic growth of size-selected ammonium sulphate particles. The RH needed to grow the particles to the measured sizes was inferred from Köhler theory. From these measurements, an uncertainty range for RHs adjusted in LACIS was obtained. LACIS measured hygroscopic growth for RHs between $90 \%$ and $99 \%$. More details about LACIS calibrations and typical uncertainty ranges can be found in $[32,38]$. 
In the second aerosol line, the aerosol coming from the DMA was diluted with $1 \mathrm{Lpm}$ dry particle-free air. From the resulting $2 \mathrm{Lpm}, 1 \mathrm{Lpm}$ was fed into the CPC and a CCNc (Cloud Condensation Nuclei counter, [40], Droplet Measurement Technologies, two column version), each. The size at the DMA was scanned to obtain a number size distribution in the range from 25 to $500 \mathrm{~nm}$, measuring at 40 different dry diameters with a logarithmic equidistant spacing. The DMA selected one size for $50 \mathrm{~s}$ before selecting the next one. The CCNc kept a constant supersaturation during the scan of the whole size distribution. To obtain the activated fraction from $\mathrm{CPC}$ and $\mathrm{CCNc}$ data, the data measured during the first $30 \mathrm{~s}$ after the change of the size at the DMA were discarded and the following $10 \mathrm{~s}$ of data were used. The $\mathrm{CCNc}$ was adjusted to supersaturations $s$ between $0.1 \%$ and $0.4 \%\left(s=S_{d}-1\right)$. The calibration curve of the $\mathrm{CCNc}$ was determined prior to the measurements for $s$ ranging from $0.1 \%$ to $0.7 \%$. For this, the activation of sizesegregated ammonium sulphate particles was measured, and the respective $s$ was inferred via Köhler theory, giving the relation between $s$ and the instrument settings, that is, the calibration curve. This curve was kept constant during the experiments, and tests of this calibration were done before, during and after the experiment period by repeatedly measuring again the activation of size-segregated ammonium sulphate particles. These repeated measurements were used to derive an uncertainty range for $s$ of the CCNc. Typical uncertainty ranges that were found for the $\mathrm{CCNc}$ were already given in [32].

In this study, also data obtained from measurements of the hygroscopic particle growth conducted with a Humidity Tandem Differential Mobility Analyzer (H-TDMA, University of Manchester) and the same marine aerosol generator will be used [41]. For a description of the H-TDMA instrument see Fuentes et al. [30].

\section{Shape Factor}

It has long been known that dry $\mathrm{NaCl}$ particles are not spherical [42]. A nonspherical shape has also been observed for particles generated from seawater samples that contained $\mathrm{NaCl}$ as the main component [27]. When particles are not spherical, a shape correction has to be accounted for to obtain a volume equivalent diameter of the particles (which is one of the parameters in the Köhler theory) from the mobility diameter selected by the DMA. The shape of particles does, however, also depend on the particle generation and on the subsequent drying process (see e.g., $[30,43])$.

Therefore, to obtain the shape factor valid for the particles generated in this study, we particularly examined particles produced from artificial seawater and from the Chaet Mix sample. Particles of different dry sizes were selected by the DMA and their sizes were measured in LACIS, which was operated at dry conditions $(<5 \% \mathrm{RH})$ for these measurements. For the evaluation of the OPS signals, a refractive index of 1.55 was used. This value was obtained using a volume mixing rule and the particle composition as

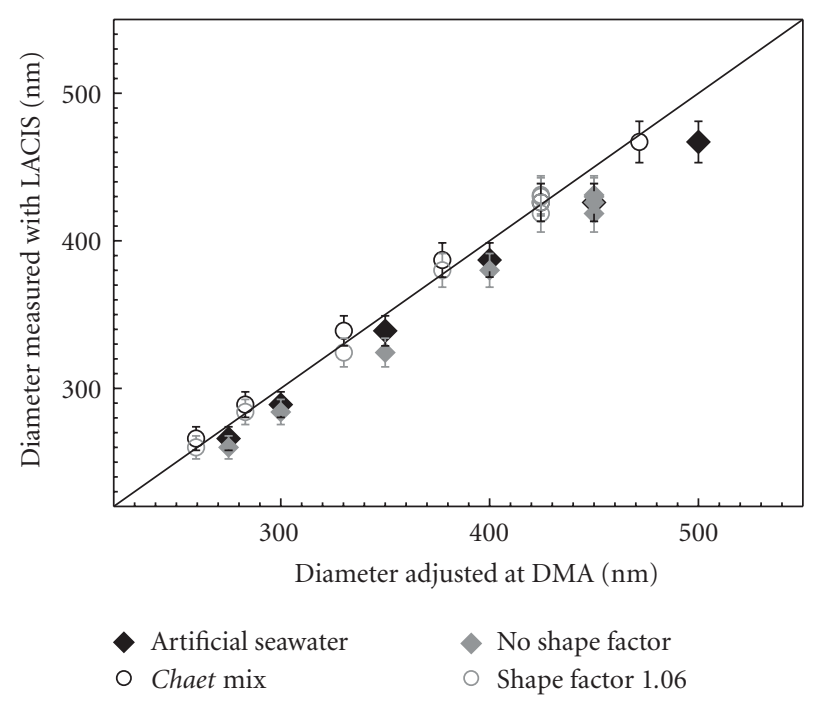

Figure 2: Comparison of dry particle diameters as selected by the DMA with the respective values measured with LACIS. The black line is the 1-to-1 line.

given in Section 3. (This value for the refractive index was still valid if an enhancement of the organic fraction due to the particle generation [41] was accounted for.) A comparison of diameters measured by the OPS with those selected by the DMA can be seen in Figure 2. When no shape factor is used, the diameters selected by the DMA are larger than those obtained from the LACIS measurement for both examined samples. The best agreement between the data and the 1-to-1 line (also shown in Figure 2) was obtained, when assuming a shape factor of 1.06. This value of 1.06 was used to calculate the volume equivalent diameters from the mass equivalent diameters for all particles generated in this study.

\section{Results and Discussion}

In Figure 3 we show measured hygroscopic growth factors and critical diameters for activation of the different samples. It is difficult to distinguish between the data obtained for the different samples, but Figure 3 serves to show that all the samples had a comparably similar behaviour with respect to hygroscopic growth and activation. For comparison, the theoretical values for sodium chloride and ammonium sulphate were added as well. The ammonium sulphate curves are indicated by the light grey lines. The sodium chloride curves are displayed as dark grey lines surrounded by grey shaded areas, where the shaded areas indicate the range of measurement uncertainties in water vapor saturation (for LACIS: in RH (i.e., $a_{w}$ ) and for the CCNc in $s$ ). These ranges correspond to uncertainties of $2 \sigma$ (i.e., the $95 \%$ confidence range) and were obtained from repeated measurements with particles from a well-known substance (ammonium sulphate), as described in Section 4.

Measured values shown for artificial seawater in Figure 3 stand out slightly from the others, as particles generated from this sample showed the largest hygroscopic growth, 


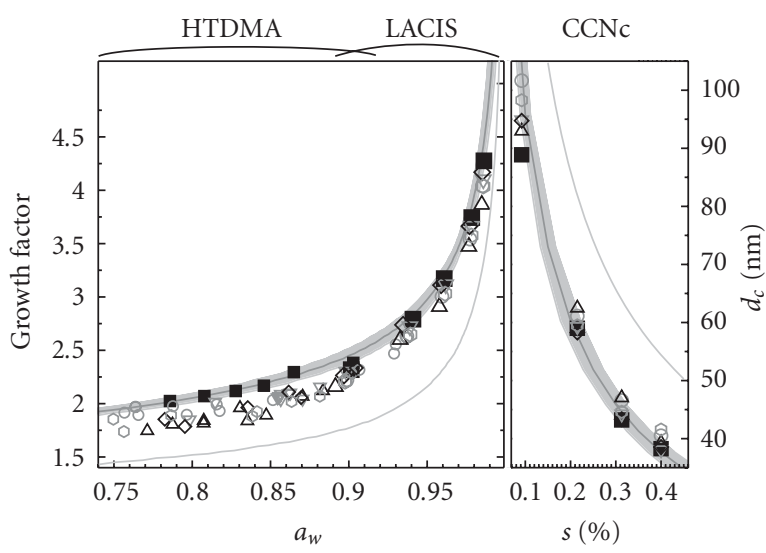

- Artificial seawater $\Delta$ Chaet mix

- Algea medium $\quad \nabla$ E. huxleyi mix

$\checkmark \mathrm{NaCI} \quad \diamond$ Phaeo mix

$-\left(\mathrm{NH}_{4}\right)_{2} \mathrm{SO}_{4} \bigcirc$ T. rotula mix

FIGURE 3: Left panel: Measured hygroscopic growth factors from the H-TDMA (smaller symbols) and LACIS (larger symbols). Right panel: Measured $d_{c}$ from the CCNc. Additional curves in both panels show the theoretical curves for sodium chloride and ammonium sulphate.

consistent with showing the lowest critical diameters for activation, $d_{c}$. These values are close to those for sodium chloride for both hygroscopic growth and activation. The theoretical data for ammonium sulphate particles clearly separate from all others, with their hygroscopic growth being smaller and their activation occurring at larger values for $d_{c}$ than for particles of any of the examined samples.

Measurements with the H-TDMA and LACIS were conducted at different dry particle diameters $(240 \mathrm{~nm}$ or $200 \mathrm{~nm}$ selected by the DMA, that is, volume equivalent diameters, $d_{p}$, of $226 \mathrm{~nm}$ or $188 \mathrm{~nm}$ when including the shape factor correction for H-TDMA and LACIS, respectively), so we show hygroscopic growth factors $\left(=d_{d} / d_{p}\right)$ as a function of water activity $a_{w}$ (i.e., of $\left.S_{d} / K=(\mathrm{RH} / 100) / K\right)$. The ranges of $a_{w}$ in which data from the H-TDMA and LACIS are shown are indicated at the top of Figure 3. The further examination of the data, which will be shown next, gives a clearer picture of the overlap between data from the two different instruments. The critical dry diameters for activation, $d_{c}$, as shown in the right panel of Figure 3, were derived from $\mathrm{CCN}$ c measurements and were also shape factor corrected with the above derived value of 1.06. While in general the data measured for hygroscopic growth and activation of the artificial seawater sample were close to those of pure $\mathrm{NaCl}$ (within measurement uncertainty), $d_{c}$ measured at $s=0.1 \%$ was lower than that of $\mathrm{NaCl}$, that is, it seemed to be easier to activate and differed from that of $\mathrm{NaCl}$ by more than the measurement uncertainty. As $\mathrm{NaCl}$ is the most hygroscopic compound in the artificial seawater, this result might indicate a problem with the CCNc at $s=0.1 \%$. During the calibration of the CCNc with sizeselected ammonium sulphate particles, a deviation of the linear relationship between the temperature gradient in the
CCNc column and the supersaturation was observed at $s=$ $0.1 \%$. The supersaturation at this lowest value likely was larger than adjusted, but could not be determined with a high accuracy. Therefore, although the values for $\rho_{\text {ion }}$ were calculated from these data points, they were not considered in the further data evaluation.

For the calculation of $\rho_{\text {ion }}$ from the measurements, a value for the surface tension $\sigma_{s}$ was needed. In [41] it is described in detail how, based on measurements, surface tension isotherms were determined for the algal exudates that were also used to prepare the samples in our study. From this, $\sigma_{s}$ of droplet solutions at the point of activation can be calculated. Droplets activating at smaller particles (and larger supersaturations) have more concentrated solutions at the point of activation, hence, smaller particles show a larger decrease in $\sigma_{s}$, if surface active compounds are present. The smallest particles for which activation was measured in our study had diameters of about $40 \mathrm{~nm}$, for which a surface tension reduction at the point of activation for the different algal exudate mixtures was found in the range from 0.5 to $3 \%$, where the largest reduction was observed for Chaet Mix [41]. For particles with sizes $>100 \mathrm{~nm}$, this reduction was $<1 \%$. Also, particle hygroscopic growth for $\mathrm{RHs}<95 \%$ is insensitive to $\sigma_{s}$ [25]. Therefore, in the present study, we used the surface tension of water for the determination of $\rho_{\text {ion }}$ in our study. As surface tension depends on temperature, for data measured with the CCNc care was taken to account for the temperature in the instrument.

Figure 4 shows $\rho_{\text {ion }}$ derived from the measurements through the use of (1) and (2) (for details on these calculations see e.g., [32]). Data is shown separately for the different samples. As mentioned above (Section 2), values of $\rho_{\text {ion }}$ are generally comparable to $\kappa$, and for example, $\rho_{\text {ion }}=$ $70 * 10^{3} \mathrm{~mol} / \mathrm{m}^{3}$ corresponds to a $\kappa$ value of 1.26 .

The overlap regions for data being derived from $\mathrm{H}$ TDMA and LACIS for the different samples can be seen clearly for the different samples in Figure 4. The data from the two different instruments are in agreement within measurement uncertainty. Also, no large deviations were found between $\rho_{\text {ion }}$ determined with LACIS at high RHs and those derived from CCNc measurements. As particle activation is sensitive to $\sigma_{s}$ [25], if the assumed value for $\sigma_{s}$ deviated much from the actual ones, this would show in differences of the $\rho_{\text {ion }}$ values obtained from LACIS and the CCNc data. A clear example for this can be found, for example, in [25], a study examining laboratory-generated SOA (Secondary Organic Aerosol). Such differences, however, were not observed in the study presented here.

In Figure 4, again theoretical curves were added. The dotted black line in the two upper panels shows the theoretical curve for sodium chloride. The red curve depicted in all panels is the theoretical curve for ammonium sulphate. The blue curve shows the theoretical values that would be expected based on the composition of artificial seawater, together with assuming a nonideal behaviour as that of $\mathrm{NaCl}$. To obtain the curve, $\rho_{\text {ion }}$ for fully dissolved artificial seawater was derived from the mass fractions of the different components contained in the sample (see Section 3), together with using a volume mixing rule 


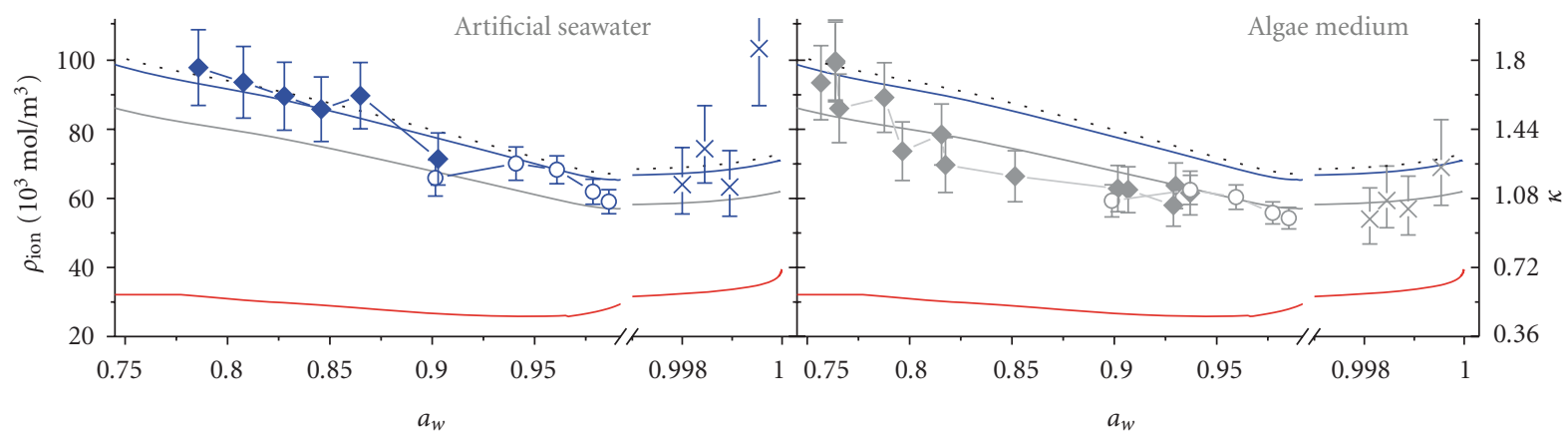

(a)

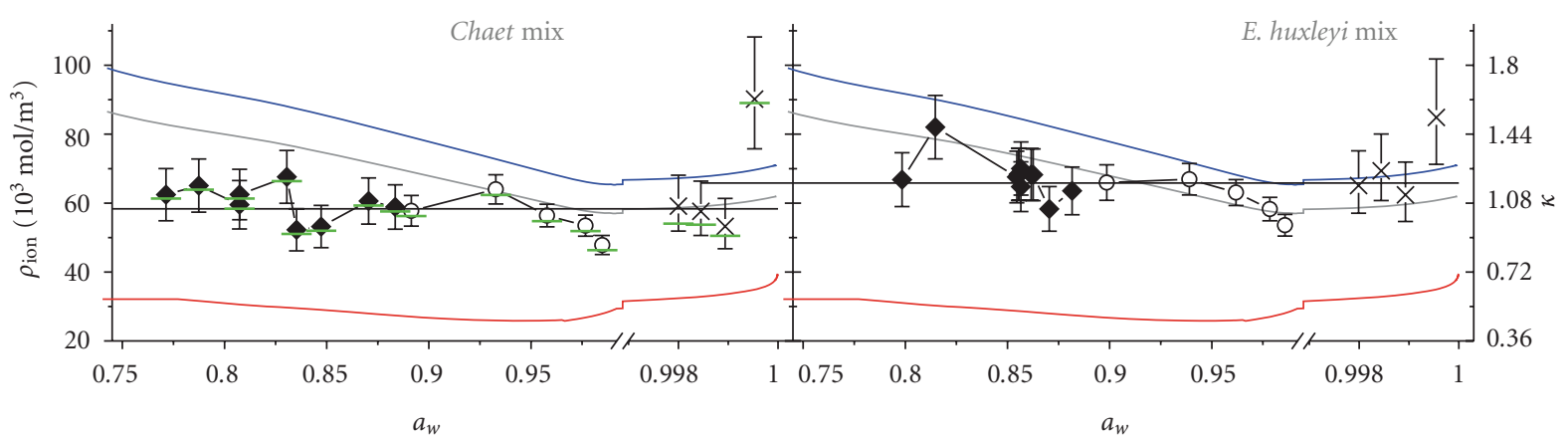

(b)

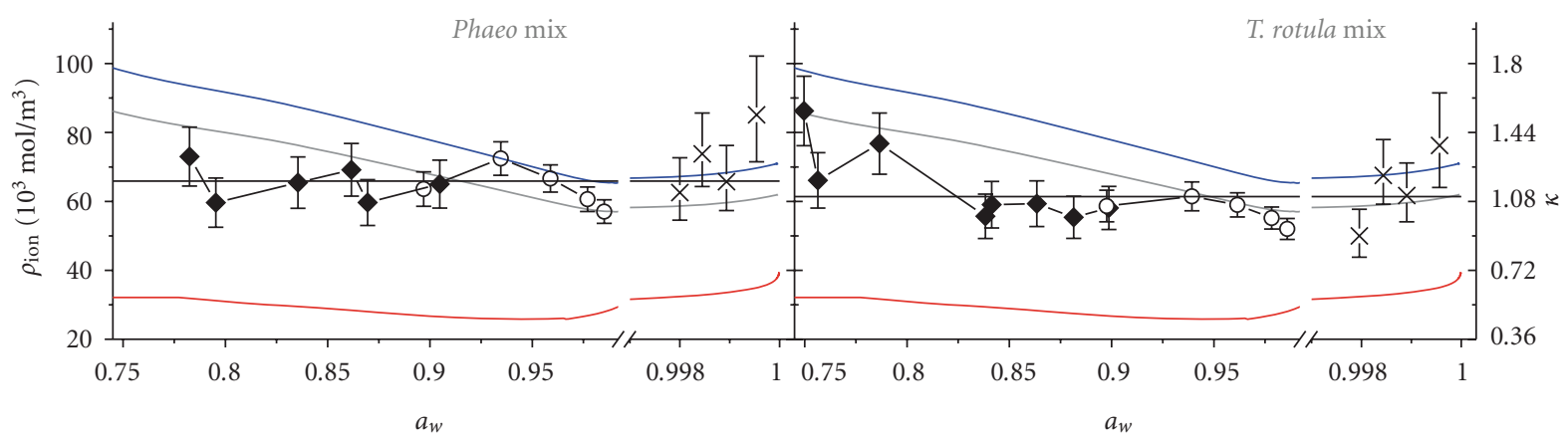

(c)

FIGURE 4: $\rho_{\text {ion }}$ derived from measured hygroscopic growth and activation; filled and open symbols were derived from H-TDMA and LACIS measurements, respectively, crosses from CCNc measurements (respective $\kappa$ values are indicated at the right axis). Additional curves are thought as guide for the eye. More details about them are given in the text.

and the properties $\left(\rho_{s}, M_{s}, \nu\right)$ of the respective components. This yielded a value of $72.3 * 10^{3} \mathrm{~mol} / \mathrm{m}^{3}$ (as compared to $74.1 * 10^{3} \mathrm{~mol} / \mathrm{m}^{3}$ for fully dissolved $\mathrm{NaCl}$ ). This value for the fully dissolved solution was then overlain with the same nonideal behaviour that is described for pure $\mathrm{NaCl}$ (see [26]), the main component of the artificial seawater. When comparing this blue curve with the $\rho_{\text {ion }}$ values derived from the measurements for the artificial seawater (blue symbols in the upper panel on the left side of Figure 4), we obtained an $R^{2}$-value of 0.77 . Therefore, we conclude that the artificial seawater sample shows a nonideal behaviour similar to that of $\mathrm{NaCl}$.

The grey line shown in all the panels of Figure 4 was obtained from the blue line (i.e., the artificial seawater curve) through lowering it by a constant factor. This factor was obtained by minimizing the least square value between the lowered (grey) curve and the $\rho_{\text {ion }}$ values obtained from the algae medium measurements (upper panel on the right side). The minimum least square was obtained for a curve that came from multiplying the curve for the artificial seawater with a factor of 0.872 . This corresponds to a $\rho_{\text {ion }}$ for the highly diluted algae medium sample of $63.0 * 10^{3} \mathrm{~mol} / \mathrm{m}^{3}$. This lowering of $\rho_{\text {ion }}$ by $12.8 \%$ compared to that of artificial seawater can be attributed to the additives that were added to the seawater to produce the algae medium. The $R^{2}$-value between the lowered (grey) curve and the measured values is 0.78 . This degree of agreement between the grey curve and $\rho_{\text {ion }}$ for the algae medium shows that a nonideal behaviour close to that of $\mathrm{NaCl}$ was also observed for this sample.

For the examined algal exudate samples, the blue and grey lines described above are also shown for comparison in 
the different panels of Figure 4. However, for all of these samples, additional horizontal straight black lines are given in the panels. These lines indicate the average $\rho_{\text {ion }}$ from the data shown in the respective panel. The values are 58.3, 65.9, 65.8, and $61.3 * 10^{3} \mathrm{~mol} / \mathrm{m}^{3}$ for Chaet Mix, E. huxleyi Mix, Phaeo Mix, and T. rotula Mix, respectively. For highly diluted solutions, these average $\rho_{\text {ion }}$ values for the algal exudate samples agree with that of the algae medium within measurement uncertainty. The components with lower hygroscopicities contained in the algae medium (i.e., the nutrients) were different to those in the algal exudate samples. The algal exudate samples will not contain substantial nutrient levels, as these were consumed by the algae during growth. Therefore, it is merely coincidental that $\rho_{\text {ion }}$ values from the algae medium are similar to those of the algal exudate samples. But this will be used later in our study to compare the concentrationdependent $\rho_{\text {ion }}$ found for the algae medium to the $\rho_{\text {ion }}$ values obtained for the algal exudate samples.

For Chaet Mix, green bars in Figure 4 indicate $\rho_{\text {ion }}$ determined using the concentration-dependent surface tension of Fuentes et al. [41], as opposed to the surface tension of water. This leads to a lowering of the average $\rho_{\text {ion }}$ of about $3 \%$ (to $\left.56.5 * 10^{3} \mathrm{~mol} / \mathrm{m}^{3}\right)$. As Chaet Mix showed the largest surface tension reduction, the respective effect for the other algal exudate samples would be even smaller. The trend of $\rho_{\text {ion }}$ with respect to the changing solution concentration is not affected.

Our measurements can be compared to those done by Sellegri et al. [44], where hygroscopic growth at $84 \% \mathrm{RH}$ was measured for particles with a dry diameter of $85 \mathrm{~nm}$. Particles were produced by bubble bursting from artificial seawater (a mixture of purely inorganic salts) and natural seawater (containing organic matter). $\rho_{\text {ion }}$ values that can be derived from their measurements are $72.2 * 10^{3} \mathrm{~mol} / \mathrm{m}^{3}$ for the artificial seawater (as compared to $86.1 * 10^{3} \mathrm{~mol} / \mathrm{m}^{3}$ at $84 \% \mathrm{RH}$ in our study) and $61.1 * 10^{3} \mathrm{~mol} / \mathrm{m}^{3}$ for the natural seawater. While this value for the natural seawater is within those obtained for our algal exudate samples, the hygroscopicity obtained for the artificial seawater in our study exceeds that given by Sellegri et al. [44] by almost $20 \%$. This difference, however, could originate in different concentrations of the salts in the artificial seawater, which is difficult to test as the concentrations used by Sellegri et al. [44] are not given in their publication. Sellegri et al. [44] also measured hygroscopic growth for ambient marine particles at the western coast of Ireland (Mace Head) and found growth factors that were still lower than those of laboratorygenerated particles from natural seawater, corresponding to $\rho_{\text {ion }}$ of $52.2 * 10^{3} \mathrm{~mol} / \mathrm{m}^{3}$.

Recently, Wex et al. [3] derived average particle hygroscopicities from collections of H-TDMA data. The data had been measured around the globe during more than a decade and were collected in overview papers by Kandler and Schütz [45] and Swietlicki et al. [46]. Wex et al. [3] give hygroscopicities in terms of the parameter $\kappa$, with $\kappa$ for the more hygroscopic particle fraction being around 0.3 for continental and 0.45 for marine aerosol, and with $\kappa$ being 0.95 in an additional (small) sea-salt mode for the marine case. These values correspond to $\rho_{\text {ion }}$ of $16.7,25.0$, and $52.8 * 10^{3} \mathrm{~mol} / \mathrm{m}^{3}$, respectively. The hygroscopicity derived from ambient data in Sellegri et al. [44] agrees well with that obtained for the average sea-salt mode in [3]. However, these values are still about $20 \%$ smaller than those measured for laboratorygenerated particles from natural seawater or from the algal exudate samples examined in this study. This might be a hint towards difficulties in imitating marine aerosol in the laboratory, which possibly can be due to the addition of particulate organic matter from the gas phase in the marine atmosphere.

We now get back to the analysis of the data shown in Figure 4 . The ability of the grey curve to represent the $\rho_{\text {ion }}$ values obtained for the algal exudate samples was evaluated. For all four samples, $R^{2}<0.5$ was obtained (with $R^{2}$ as low as 0.03 for Phaeo Mix). The variances between the $\rho_{\text {ion }}$ values and the grey curve were larger than those between the $\rho_{\text {ion }}$ values and the average $\rho_{\text {ion }}$ by a factor of $6.8,1.4,3.6$, and 1.6 for Chaet Mix, E. huxleyi Mix, Phaeo Mix, and T. rotula Mix, respectively. From both, the $R^{2}$-values and the variances, it follows that using a constant, average $\rho_{\text {ion }}$ (i.e., independent of the solution concentration) describes the data better than using the grey curve. The addition of the algal exudates seems to cause the nonideal behaviour of the solutions to become independent of the solution concentration.

We also examined a possible influence of kinetic effects on the measured hygroscopic growth. Data were obtained from the two LACIS-OPCs that were positioned after the first meter of the flow tube and at its end after 7 meters. These positions correspond to residence times of the particles in the hydrated state of about 3 and 10 seconds. Figure 5 shows the measured hygroscopic growth factors. The grey area indicates the uncertainty in $a_{w}$ for the measurements done in the upper part of the flow tube $(2 \sigma$, i.e., $95 \%$ confidence range), the error bars indicate the same for the measurements done at the end of the flow tube. No noticeable difference between the measured values after 3 and after 10 seconds can be seen. Therefore, in the data presented here, we do not see an influence of kinetics on the hygroscopic growth behaviour of the examined samples for the examined time scale of 3 to 10 seconds.

Summarizing we can say that rather than complicating, the addition of algal exudates to sea-salt particles seems to simplify the description of their hygroscopic behaviour. Hygroscopic growth and activation could be described by using values for $\sigma_{s}$ and $\rho_{\text {ion }}$ that were independent of the solution concentration over the wide range of concentrations examined in this study. This is consistent with no substantial effect of surface tension and a constant nonideality with respect to solute concentration. This, however, is contrary to well-established behaviour of $\mathrm{NaCl}$ [26] and sea-salt solutions (e.g., [27], and artificial seawater examined in our study). It is possible that this simplification in the hygroscopic behaviour could be caused by cancellation of effects that are/could be concentration dependent, such as nonideality, shape factor, stepwise deliquescence, or surface tension (where it has to be repeated that hygroscopic growth for RHs $<95 \%$ has been shown to be insensitive to surface tension, [25]). Overall, the limited data set that was examined in this study does not allow to examine the underlying effects in more detail. 


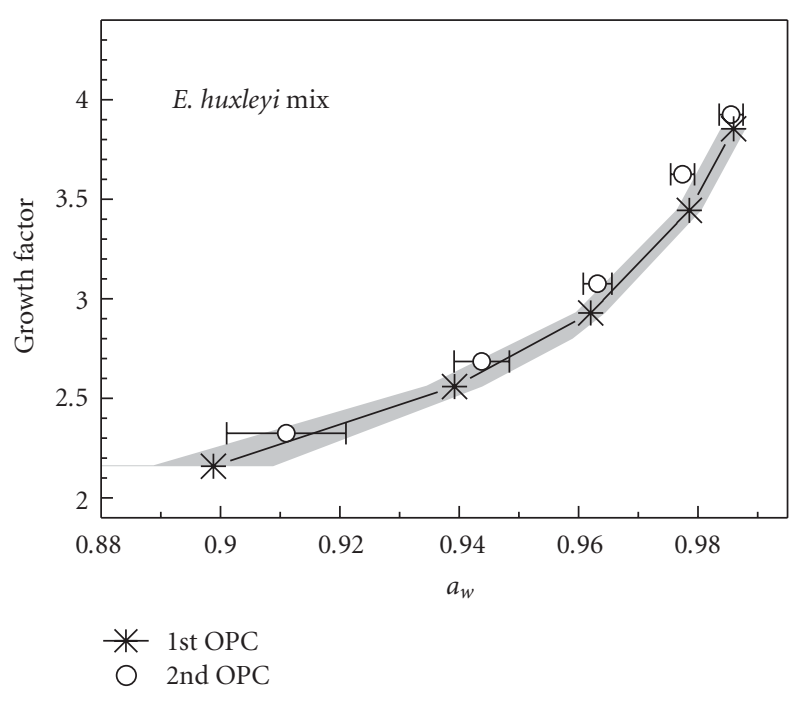

(a)

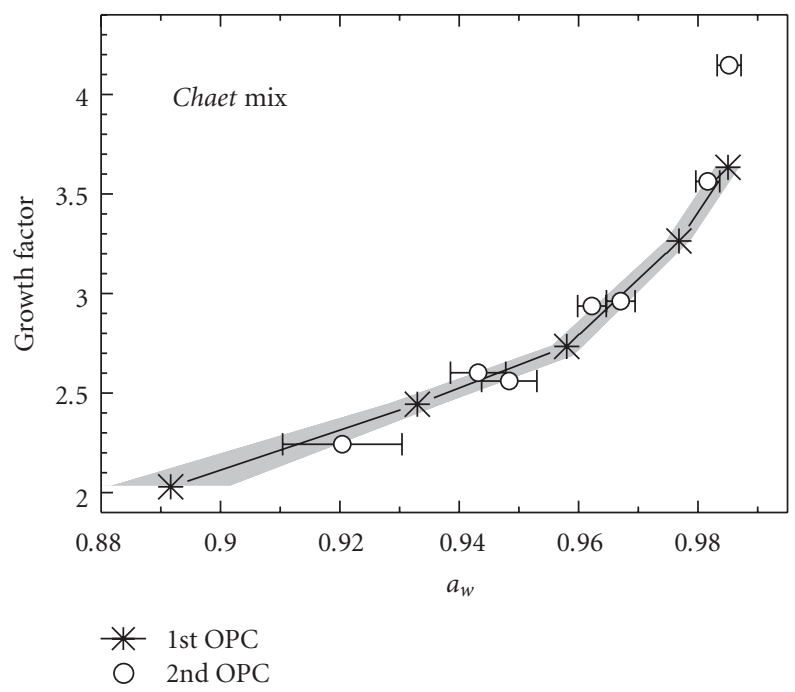

(b)

FIgure 5: Comparison of hygroscopic growth measured with LACIS at two different positions of the LACIS flow tube, that is, after different residence times of 3 and 10 seconds.

The independence of $\sigma_{s}$ and $\rho_{\text {ion }}$ from the solution concentration enables the description of the particle hygroscopicity with a single parameter, be it $\rho_{\text {ion }}$ or $\kappa$. The average $\rho_{\text {ion }}$ shown in in Figure 4 corresponds to $\kappa$ of $1.05,1.19$, 1.19 , and 1.10. When the hygroscopic growth factors of, for example, a $100 \mathrm{~nm}$ particle at $90 \% \mathrm{RH}$ is calculated from these average $\rho_{\text {ion }}$ values, it is found to range from 2.15 to 2.24 . These growth factors are slightly larger than those given by McFiggans et al. [47] for atmospheric marine particles, which range from 2.06 to 2.14 for the above mentioned particle size and RH. This discrepancy, however, can be attributed to additional particulate matter that can be acquired by marine particles, for example, from the gas phase. Among the possible candidates are non-sea-salt sulphates from Dimethylsulfide (DMS, [48]) or additional SOA mass from biogenic sources [13], but also secondary aerosol mass originating from sources as the terrestrial biosphere or from anthropogenic origin that are transported over the ocean [14]. Therefore, as, due to its vast size, a complete marine environment can not be imitated, our focus here was on a subsystem, that is, on the influence of algal exudates on hygroscopic growth and activation, for which it was found that a simple description is possible.

\section{Summary and Conclusion}

In the present study, an examination of the influence of algal exudate, that is, of biogenic organics, in seawater samples on hygroscopic growth and droplet activation behaviour of particles produced from the samples was introduced. The algal exudates were obtained from culturing four different phytoplankton species and subsequent removal of the cell biomass by filtration. Algal exudate was produced from either Chaetoceros sp., Emiliana huxleyi, Phaeocystis, and Thalassiosira rotula, which are all major contributors to primary production of organic matter in the ocean. Mixtures of these exudates were added to seawater, yielding seawater proxies with concentrations of dissolved organic carbon on the order of those expected in regions of high biological activity. These, thus, prepared samples were examined, together with a sample of artificial seawater (that contained no organics) and with algae medium, which was the initial natural seawater sample with added nutrients employed for growing the algal cultures. The hygroscopic growth of samples containing algal exudate was measured twice, once after 3 and once after 10 seconds of residence time at humidified conditions. No change in the measured hygroscopic growth was detected, that is, no kinetic effects could be observed in this time range.

Hygroscopic growth and particle activation of the algal exudate samples and of the algae medium were found to be reduced by less than $10 \%$, compared to artificial seawater, and they were clearly above that of ammonium sulphate. For the determination of the hygroscopicity parameter of the samples, the surface tension of water was used. Concentration-dependent nonideal behaviour was observed for the artificial seawater and for the algae medium sample, following that described for $\mathrm{NaCl}$ [26]. This concentration dependence was not observed for the samples containing algal exudate. Therefore, it is possible to use a single parameter description of the hygroscopicity of the particles generated from the algal exudate samples, independent of the concentration of inorganic and organic solutes in the particle or droplet solution, with parameters as, for example, $\rho_{\text {ion }}[31]$ or $\kappa[4]$.

Overall, the examined algal exudates in the samples did not complicate the description of the hygroscopic behaviour of particles generated from these samples but rather made it more simple. It should be stressed, however, that marine particles can contain additional matter acquired from the gas phase, where marine sources can add inorganic matter (e.g., non-sea-salt sulphates from Dimethylsulfide, [48]) and organic matter [13], and to which also anthropogenic sources and the terrestrial biosphere can contribute. 


\section{Acknowledgments}

This work was performed as part of EUROCHAMPII and also supported by the UK Natural Environment Research Council (Grant numbers NE/D005175/1 and NE/G000247/1). G. Tsagkogeorgas was funded by a Marie Curie Initial Training Network [CLOUD-ITN, No 215072]. The authors thank Ottmar Möhler from the KIT in Karlsruhe, Germany, for providing the CCNc.

\section{References}

[1] C. D. O’Dowd and G. de Leeuw, "Marine aerosol production: a review of the current knowledge," Philosophical Transactions of the Royal Society A, vol. 365, no. 1856, pp. 1753-1774, 2007.

[2] C. Pilinis, S. N. Pandis, and J. H. Seinfeld, "Sensitivity of direct climate forcing by atmospheric aerosols to aerosol size and composition," Journal of Geophysical Research, vol. 100, no. 9, pp. 18-754, 1995.

[3] H. Wex, G. McFiggans, S. Henning, and F. Stratmann, "Influence of the external mixing state of atmospheric aerosol on derived CCN number concentrations," Geophysical Research Letters, vol. 37, no. 10, Article ID L10805, 2010.

[4] M. D. Petters and S. M. Kreidenweis, "A single parameter representation of hygroscopic growth and cloud condensation nucleus activity," Atmospheric Chemistry and Physics, vol. 7, no. 8, pp. 1961-1971, 2007.

[5] A. H. Woodcock, C. F. Kientzler, A. B. Arons, and D. C. Blanchard, "Giant condensation nuclei from bursting bubbles," Nature, vol. 172, no. 4390, pp. 1144-1145, 1953.

[6] G. Feingold, W. R. Cotton, S. M. Kreidenweis, and J. T. Davis, "The impact of giant cloud condensation nuclei on drizzle formation in stratocumulus: implications for cloud radiative properties," Journal of the Atmospheric Sciences, vol. 56, no. 24, pp. 4100-4117, 1999.

[7] A. M. Blyth, S. G. Lasher-Trapp, W. A. Cooper, C. A. Knight, and J. Latham, "The role of giant and ultragiant nuclei in the formation of early radar echoes in warm cumulus clouds," Journal of the Atmospheric Sciences, vol. 60, no. 21, pp. 2557$2572,2003$.

[8] H. E. Gerber, G. M. Frick, J. B. Jensen, and J. G. Hudson, "Entrainment, mixing, and microphysics in trade-windcumulus," Journal of the Meteorological Society of Japan, vol. 86A, pp. 87-106, 2008.

[9] C. Textor, M. Schulz, S. Guibert et al., "Analysis and quantification of the diversities of aerosol life cycles within AeroCom," Atmospheric Chemistry and Physics, vol. 6, no. 7, pp. 1777$1813,2006$.

[10] D. A. Randell, J. A. Coakley Jr., C. W. Fairall, R. A. Kropfli, and D. H. Lenschow, "Outlook for research on subtropical marine stratiform clouds," Bulletin of the American Meteorological Society, vol. 65, no. 12, pp. 1290-1301, 1984.

[11] B. A. Albrecht, "Aerosols, cloud microphysics, and fractional cloudiness," Science, vol. 245, no. 4923, pp. 1227-1230, 1989.

[12] L. I. Aluwihare and D. J. Repeta, "A comparison of the chemical characteristics of oceanic DOM and extracellular DOM produced by marine algae," Marine Ecology Progress Series, vol. 186, pp. 105-117, 1999.

[13] N. Meskhidze and A. Nenes, "Phytoplankton and cloudiness in the southern ocean," Science, vol. 314, no. 5804, pp. 1419$1423,2006$.

[14] E. G. Stephanou, "Analysis of anthropogenic and biogenic lipids in the aerosol of a coastal area in East Mediterranean
Sea," Fresenius' Journal of Analytical Chemistry, vol. 339, no. 10, pp. 780-784, 1991.

[15] C. A. Randles, L. M. Russell, and V. Ramaswamy, "Hygroscopic and optical properties of organic sea salt aerosol and consequences for climate forcing," Geophysical Research Letters, vol. 31, no. 16, Article ID L16108, 2004.

[16] F. Cavalli, M. C. Facchini, S. Decesari et al., "Advances in characterization of size-resolved organic matter in marine aerosol over the North Atlantic," Journal of Geophysical Research D, vol. 109, no. 24, Article ID D24215, 2004.

[17] C. D. O’Dowd, M. C. Facchini, F. Cavalli et al., "Biogenically driven organic contribution to marine aerosol," Nature, vol. 431, no. 7009, pp. 676-680, 2004.

[18] M. Mochida, Y. Kitamori, K. Kawamura, Y. Nojiri, and K. Suzuki, "Fatty acids in the marine atmosphere: factors governing their concentrations and evaluation of organic films on sea-salt particles," Journal of Geophysical Research D, vol. 107, no. 17, article 4325, 2002.

[19] H. Tervahattu, K. Hartonen, V.-M. Kerminen et al., "New evidence of an organic layer on marine aerosols," Journal of Geophysical Research D, vol. 107, no. 7, article 4053, 2002.

[20] H. Tervahattu, J. Juhanoja, and K. Kupiainen, "Identification of an organic coating on marine aerosol particles by TOFSIMS," Journal of Geophysical Research D, vol. 107, no. 16, article 4319, 2002.

[21] G. B. Ellison, A. F. Tuck, and V. Vaida, "Atmospheric processing of organic aerosols," Journal of Geophysical Research $D$, vol. 104, no. 9, pp. 11633-11641, 1999.

[22] M. C. Facchini, M. Mircea, S. Fuzzi, and R. J. Charlson, "Cloud albedo enhancement by surface-active organic solutes in growing droplets," Nature, vol. 401, no. 6750, pp. 257-259, 1999.

[23] R. H. Moore, E. D. Ingall, A. Sorooshian, and A. Nenes, "Molar mass, surface tension, and droplet growth kinetics of marine organics from measurements of CCN activity," Geophysical Research Letters, vol. 35, no. 7, Article ID L07801, 2008.

[24] K. Sellegri, C. D. O'Dowd, Y. J. Yoon, S. G. Jennings, and G. de Leeuw, "Surfactants and submicron sea spray generation," Journal of Geophysical Research D, vol. 111, no. 22, Article ID D22215, 2006.

[25] H. Wex, F. Stratmann, D. Topping, and G. McFiggans, "The Kelvin versus the raoult term in the köhler equation," Journal of the Atmospheric Sciences, vol. 65, no. 12, pp. 4004-4016, 2008.

[26] H. R. Pruppacher and J. D. Klett, Microphysics of Clouds and Precipitation, Kluwer Academic Publishers, Dordrecht, The Netherlands, 1997.

[27] D. Niedermeier, H. Wex, J. Voigtländer et al., "LACISmeasurements and parameterization of sea-salt particle hygroscopic growth and activation," Atmospheric Chemistry and Physics, vol. 8, no. 3, pp. 579-590, 2008.

[28] C. W. Brown and J. A. Yoder, "Coccolithophorid blooms in the global ocean," Journal of Geophysical Research, vol. 99, no. 4, pp. 7467-7482, 1994.

[29] V. Schoemann, S. Becquevort, J. Stefels, V. Rousseau, and C. Lancelot, "Phaeocystis blooms in the global ocean and their controlling mechanisms: a review," Journal of Sea Research, vol. 53, no. 1-2, pp. 43-66, 2005.

[30] E. Fuentes, H. Coe, D. Green, G. De Leeuw, and G. McFiggans, "Laboratory-generated primary marine aerosol via bubblebursting and atomization," Atmospheric Measurement Techniques, vol. 3, pp. 1-22, 2010.

[31] H. Wex, T. Hennig, I. Salma et al., "Hygroscopic growth and measured and modeled critical super-saturations of an 
atmospheric HULIS sample," Geophysical Research Letters, vol. 34, no. 2, Article ID L02818, 2007.

[32] H. Wex, M. D. Petters, C. M. Carrico et al., "Towards closing the gap between hygroscopic growth and activation for secondary organic aerosol-part 1: evidence from measurements," Atmospheric Chemistry and Physics, vol. 9, no. 12, pp. 3987-3997, 2009.

[33] D. Kester, I. Duedall, D. Conners, and R. Pytkowicz, "Preparation of artificial seawater," Limnology and Oceanography, vol. 12, pp. 176-179, 1976.

[34] R. Guillard, "Culture of phytoplankton for feeding marine invertebrates," in Culture of Marine Invertebrate Animals, pp. 26-60, Plenum Press, New York, NY, USA, 1975.

[35] V. Ittekkot, "Variations of dissolved organic matter during a plankton bloom: qualitative aspects, based on sugar and amino acid analyses," Marine Chemistry, vol. 11, no. 2, pp. 143-158, 1982.

[36] M. W. Lomas, P. M. Glibert, D. A. Clougherty et al., "Elevated organic nutrient ratios associated with brown tide algal blooms of Aureococcus anophagefferens (Pelagophyceae)," Journal of Plankton Research, vol. 23, no. 12, pp. 1339-1344, 2001.

[37] F. Fraga, "Phytoplanktonic biomass synthesis: application to deviations from Redfield stoichiometry," Scientia Marina, vol. 65, no. 2, pp. 153-169, 2001.

[38] H. Wex, A. Kiselev, F. Stratmann, J. Zoboki, and F. Brechtel, "Measured and modeled equilibrium sizes of $\mathrm{NaCl}$ and $\left(\mathrm{NH}_{4}\right)_{2} \mathrm{SO}_{4}$ particles at relative humidities up to $99.1 \%$," Journal of Geophysical Research D, vol. 110, no. 21, Article ID D21212, 2005.

[39] A. Kiselev, H. Wex, F. Stratmann, A. Nadeev, and D. Karpushenko, "White-light optical particle spectrometer for in situ measurements of condensational growth of aerosol particles," Applied Optics, vol. 44, no. 22, pp. 4693-4701, 2005.

[40] G. C. Roberts and A. Nenes, "A continuous-flow streamwise thermal-gradient CCN chamber for atmospheric measurements," Aerosol Science and Technology, vol. 39, no. 3, pp. 206221, 2005.

[41] E. Fuentes, H. Coe, D. Green, and G. McFiggans, "On the impacts of phytoplankton-derived organic matter on the properties of primary marine aerosol," in preparation.

[42] W. P. Kelly and P. H. McMurry, "Measurement of particle density by inertial classification of differential mobility analyzer-generated monodisperse aerosols," Aerosol Science and Technology, vol. 17, no. 3, pp. 199-212, 1992.

[43] D. Rose, S. S. Gunthe, E. Mikhailov et al., "Calibration and measurement uncertainties of a continuous-flow cloud condensation nuclei counter (DMT-CCNC): CCN activation of ammonium sulfate and sodium chloride aerosol particles in theory and experiment," Atmospheric Chemistry and Physics, vol. 8, no. 5, pp. 1153-1179, 2008.

[44] K. Sellegri, P. Villani, D. Picard, R. Dupuy, C. O’Dowd, and P. Laj, "Role of the volatile fraction of submicron marine aerosol on its hygroscopic properties," Atmospheric Research, vol. 90, no. $2-4$, pp. 272-277, 2008.

[45] K. Kandler and L. Schütz, "Climatology of the average watersoluble volume fraction of atmospheric aerosol," Atmospheric Research, vol. 83, no. 1, pp. 77-92, 2007.

[46] E. Swietlicki, H.-C. Hansson, K. Hämeri et al., "Hygroscopic properties of submicrometer atmospheric aerosol particles measured with H-TDMA instruments in various environments: a review," Tellus Series B, vol. 60, no. 3, pp. 432-469, 2008.
[47] G. McFiggans, P. Artaxo, U. Baltensperger et al., "The effect of physical and chemical aerosol properties on warm cloud droplet activation," Atmospheric Chemistry and Physics, vol. 6, no. 9, pp. 2593-2649, 2006.

[48] P. K. Quinn, D. S. Covert, T. S. Bates, V. N. Kapustin, D. C. Ramsey-Bell, and L. M. McInnes, "Dimethylsulfide/cloud condensation nuclei/climate system: relevant size-resolved measurements of the chemical and physical properties of atmospheric aerosol particles," Journal of Geophysical Research, vol. 98, no. 6, pp. 411-427, 1993. 

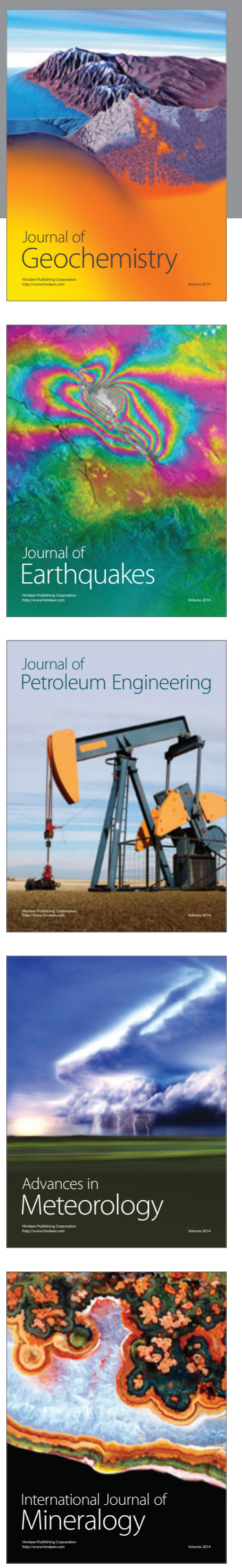
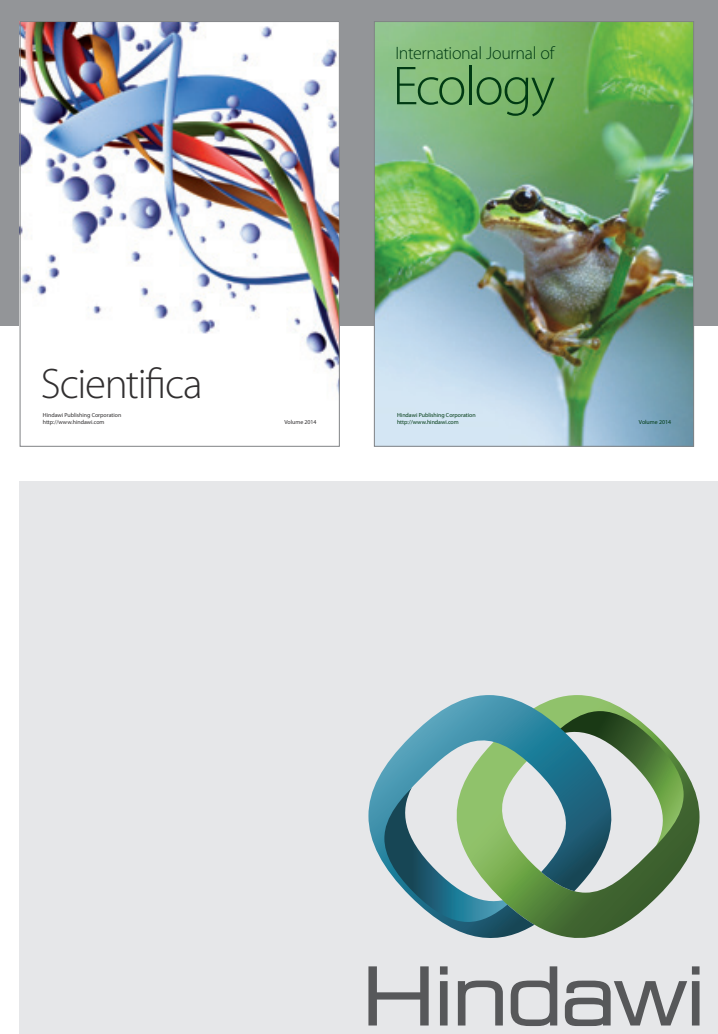

Submit your manuscripts at http://www.hindawi.com
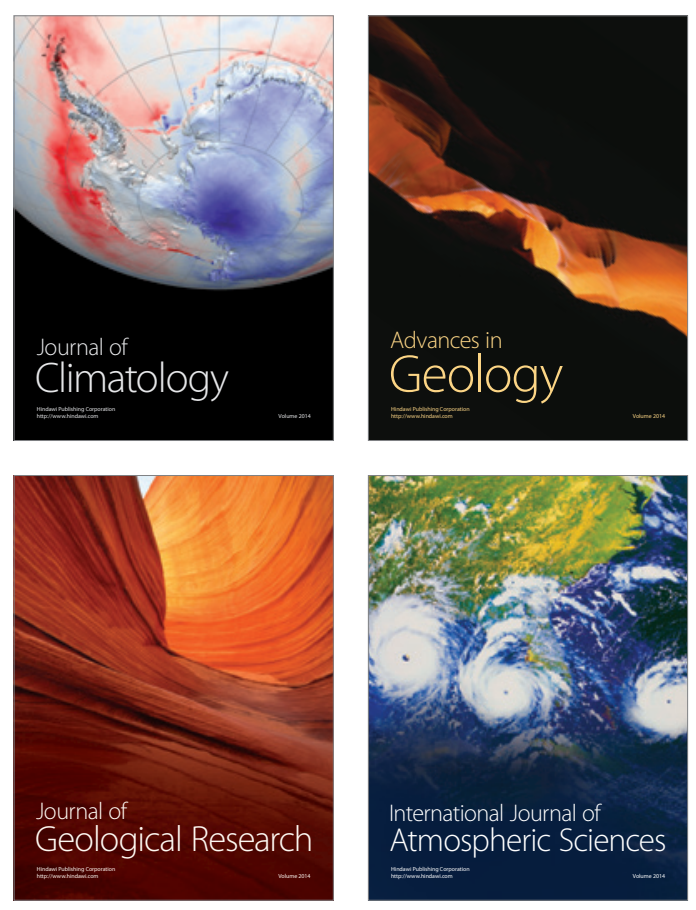
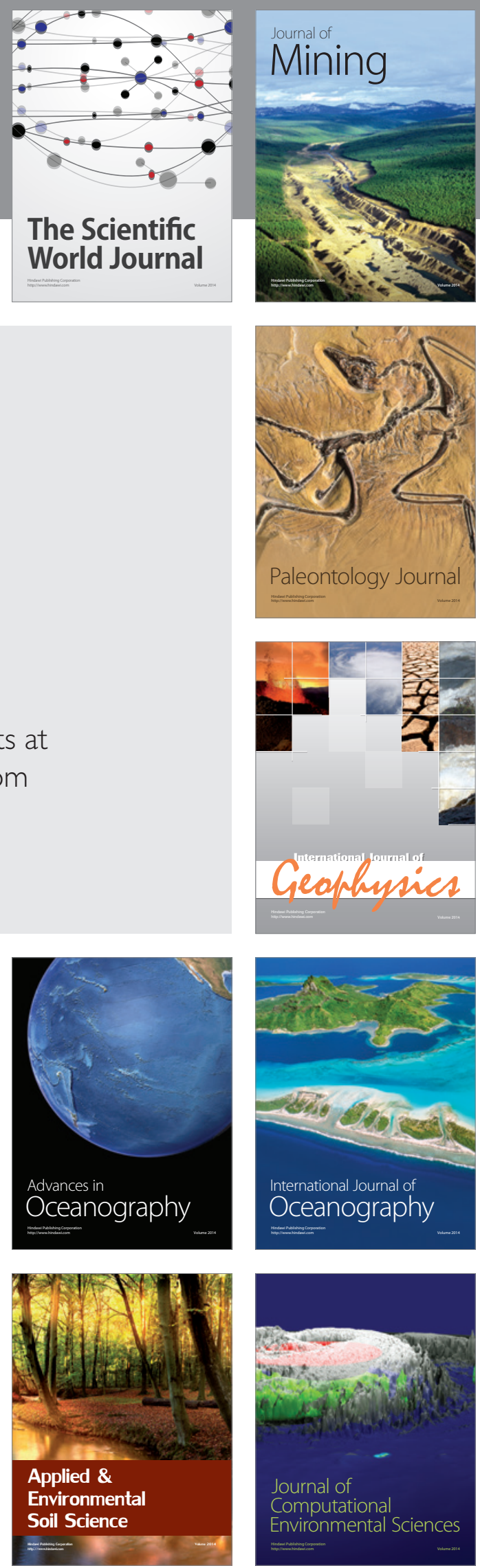